\title{
Minimal Wave Speed of Bacterial Colony Model with Saturated Functional Response
}

\author{
Tianran Zhang ${ }^{1}$ and Qingming Gou ${ }^{2}$ \\ ${ }^{1}$ School of Mathematics and Statistics, Southwest University, Chongqing 400715, China \\ ${ }^{2}$ College of Mathematics \& Computer Science, Yangtze Normal University, Chongqing 408100, China \\ Correspondence should be addressed to Tianran Zhang; zhtr0123@126.com
}

Received 12 January 2014; Accepted 24 February 2014; Published 31 March 2014

Academic Editor: Weiming Wang

Copyright (c) 2014 T. Zhang and Q. Gou. This is an open access article distributed under the Creative Commons Attribution License, which permits unrestricted use, distribution, and reproduction in any medium, provided the original work is properly cited.

\begin{abstract}
By considering bacterium death and general functional response we develop previous model of bacterial colony which focused on the traveling speed of bacteria. The minimal wave speed for our model is expressed by parameters and the necessary and sufficient conditions for traveling wave solutions (TWSs) are given. To prove the existence of TWSs, an auxiliary system is introduced and the existence of TWSs for this auxiliary system is proved by Schauder's fixed point theorem. The limit arguments show the existence of TWSs for original system. By introducing negative one-sided Laplace transform, we prove the nonexistence of TWSs.
\end{abstract}

\section{Introduction}

Experiments show that bacterial colonies on agar plates with nutrients exhibit a variety of sizes and shapes [1-7]. According to the substrate softness and nutrient concentration, the colony patterns are divided into five types $[6,8]$. Why were so many rich diffusive patterns observed in bacterial experiments? To answer this question, lots of diffusive mathematical models have been proposed and studied [4, 7, 9-16]. In these mathematical models, the colony patterns are proved or simulated on bounded domains. For bacterial colony, the colony speed is one of the most important focuses and traveling wave solution (TWS) can foresee such speed. Thus many researches studied the bacterial colony speeds through TWSs [17-24].

To more exactly anticipate the traveling speed of bacterial colony, we develop above TWS models to a more accurate bacterial colony model with bacterium death and general functional response, which is more complex compared with above TWS models. Let $N(t, x)$ and $B(t, x)$ denote the concentrations of nutrients and bacteria at time $t$ and position $x$, respectively. Then our model is as follows:

$$
\begin{aligned}
& N_{t}=d_{N} N_{x x}-f(N) B, \\
& B_{t}=d_{B} B_{x x}+\kappa f(N) B-d B,
\end{aligned}
$$

where parameters $d_{N}$ and $d_{B}$ denote the motility of the nutrients and bacteria. $\kappa$ is the conversion rate of nutrients to bacteria and $d$ is the death rate of bacteria. Function $f(N)$ is the functional response to nutrients. For simplicity, we assume $f(N)=k_{1} N /\left(1+k_{2} N\right)$ with $k_{1}>0$ and $k_{2}>0$. Actually, in the following proof we only use the monotonicity and boundedness of $f(N)$.

In this paper, the minimal wave speed $c^{*}$ is given and the necessary and sufficient conditions for the existence of TWSs are obtained. To arrive at such aim, the existence of TWSs is proved by Schauder's fixed point theorem and the nonexistence is finished by negative one-sided Laplace transform proposed firstly by us. To apply Schauder's fixed point theorem, a bounded invariant cone is needed. Such cone is constructed generally by a pair of upper and lower solutions. However, it is difficult for us to construct such solutions for model (1). Consequently, an auxiliary system is introduced, for which the upper and lower solutions can be easily constructed and are very simple. Such type of upper and lower solutions is motivated by Diekmann [25]. Then limit arguments give the existence of TWSs of model (1). Twosided Laplace transform was firstly introduced by Carr and Chmaj [26] to prove nonexistence of TWSs and was further applied by [27-29]. However, the introduction of negative one-sided Laplace transform simplifies the proof. 
This paper is organized as follows. In the next section, an auxiliary system is firstly introduced and the existence of TWSs is proved by Schauder's fixed point theorem. Then limit arguments give the existence of TWSs for original system. In Section 3, the negative one-sided Laplace transform is defined and then the nonexistence of TWSs is obtained.

\section{Existence of Traveling Wave Solution}

A traveling wave solution of system (1) is a nonnegative nontrivial solution of the form

$$
N(t, x)=U(\xi), \quad B(t, x)=V(\xi), \quad \xi=x+c t,
$$

satisfying boundary condition

$$
\begin{aligned}
& (U(-\infty), V(-\infty))=\left(N^{0}, 0\right), \\
& (U(+\infty), V(+\infty))=\left(N^{1}, 0\right),
\end{aligned}
$$

where $N^{0}>0$ is initial density of nutrients. It is obvious that $N^{0}>N^{1} \geq 0$.

Define $c^{*}=2 \sqrt{d_{B}\left[\kappa f\left(N^{0}\right)-d\right]}$. The existence of traveling wave solutions is given as follows.

Theorem 1. Suppose $f\left(N^{0}\right)>d / \kappa$. For any $c \geq c^{*}$ system (1) has a traveling wave solution $(U(x+c t), V(x+c t))$ satisfying boundary conditions (3) such that $U(\xi)$ is nonincreasing in $\mathbb{R}$ and $f\left(N^{1}\right)<d / \kappa$. Furthermore, one has that

$$
\int_{-\infty}^{+\infty} V(\eta) d \eta=\frac{\kappa c}{d}\left(N^{0}-N^{1}\right), \quad 0 \leq V(\xi) \leq \kappa\left(N^{0}-N^{1}\right)
$$

for any $\xi \in \mathbb{R}$.

Substituting wave profile $N(t, x)=U(\xi), B(t, x)=$ $V(\xi), \quad \xi=x+c t$ into system (1) yields the following equations:

$$
\begin{aligned}
& c U^{\prime}=d_{N} U^{\prime \prime}-f(U) V, \\
& c V^{\prime}=d_{B} V^{\prime \prime}+\kappa f(U) V-d V,
\end{aligned}
$$

where' denotes the derivative with respect to $\xi$.

To prove the existence of solutions of (5) satisfying (3), we construct an auxiliary system:

$$
\begin{aligned}
& c U^{\prime}=d_{N} U^{\prime \prime}-f(U) V, \\
& c V^{\prime}=d_{B} V^{\prime \prime}+\kappa f(U) V-d V-\gamma V^{2},
\end{aligned}
$$

where $\gamma$ is a positive constant and can be supposed to be small enough according to what we will need. Next, an invariant cone will be constructed and Schauder's fixed point theorem will be used to prove the existence of traveling wave solutions. We firstly linearize the second equation of $(6)$ at $\left(N^{0}, 0\right)$ and obtain

$$
c \phi^{\prime}=d_{B} \phi^{\prime \prime}+\kappa f\left(N^{0}\right) \phi-d \phi .
$$

Obviously, the characteristic equation is

$$
H(\lambda)=d_{B} \lambda^{2}-c \lambda+\kappa f\left(N^{0}\right)-d=0 .
$$

Denote $\lambda_{1}=\left(c-\sqrt{c^{2}-c^{* 2}}\right) /\left(2 d_{B}\right)$ and $\lambda_{2}=(c+$ $\left.\sqrt{c^{2}-c^{* 2}}\right) /\left(2 d_{B}\right)$. In the remainder of this section, we always suppose $\kappa f\left(N^{0}\right)>d$ and $c>c^{*}$ hold unless other conditions are specified. Define

$$
\begin{aligned}
& \underline{U}(\xi)=\max \left\{N^{0}-\sigma e^{\alpha \xi}, 0\right\}, \\
& \bar{V}(\xi)=\min \left\{e^{\lambda_{1} \xi}, V^{0}\right\} \\
& \underline{V}(\xi)=\max \left\{e^{\lambda_{1} \xi}\left(1-M e^{\varepsilon \xi}\right), 0\right\},
\end{aligned}
$$

where $V^{0}=\left(\kappa f\left(N^{0}\right)-d\right) / \gamma$ and $\gamma<\kappa f\left(N^{0}\right)-d$.

Lemma 2. The function $\bar{V}(\xi)$ satisfies inequality

$$
c \bar{V}^{\prime} \geq d_{B} \bar{V}^{\prime \prime}+\kappa f\left(N^{0}\right) \bar{V}-d \bar{V}-\gamma \bar{V}^{2},
$$

for any $\xi \neq \ln V^{0} / \lambda_{1}$

Proof. Firstly, assume $\xi<\ln V^{0} / \lambda_{1}$ and, therefore, $\bar{V}(\xi)=$ $e^{\lambda_{1} \xi}$. Since $\bar{V}(\xi)$ satisfies (7), we have

$$
c \bar{V}^{\prime}-d_{B} \bar{V}^{\prime \prime}-\kappa f\left(N^{0}\right) \bar{V}+d \bar{V}+\gamma \bar{V}^{2}=\gamma \bar{V}^{2} \geq 0 .
$$

Secondly, let $\xi>\ln V^{0} / \lambda_{1}$, which implies $\bar{V}(\xi)=V^{0}$. We have that

$$
\begin{aligned}
& c \bar{V}^{\prime}- d_{B} \bar{V}^{\prime \prime}-\kappa f\left(N^{0}\right) \bar{V}+d \bar{V}+\gamma \bar{V}^{2} \\
&=-\kappa f\left(N^{0}\right) V^{0}+d V^{0}+\gamma V^{0^{2}}=0 .
\end{aligned}
$$

The proof is completed.

Lemma 3. For $0<\alpha<\min \left\{c / d_{N}, \lambda_{1}\right\}$ and $\sigma>$ $\max \left\{N^{0}, f\left(N^{0}\right) /\left(c-d_{N} \alpha\right)\right\}$, the function $\underline{U}(\xi)$ satisfies

$$
c \underline{U}^{\prime} \leq d_{N} \underline{U}^{\prime \prime}-f(\underline{U}(\xi)) \bar{V}(\xi),
$$

for any $\xi \neq 1 / \alpha \ln \left(N^{0} / \sigma\right)$.

Proof. It is easy to show that $1 / \alpha \ln \left(N^{0} / \sigma\right)<0 \leq$ $\min \left\{0, \ln V^{0} / \lambda_{1}\right\}$. When $\xi>1 / \alpha \ln \left(N^{0} / \sigma\right)$, then $\underline{U}(\xi)=0$ and the lemma is obviously true. Now, suppose $\xi<1 / \alpha \ln \left(N^{0} / \sigma\right)$. Then $\underline{U}(\xi)=N^{0}-\sigma e^{\alpha \xi}$ and

$$
\begin{aligned}
-c \underline{U}^{\prime} & +d_{N} \underline{U}^{\prime \prime}-f(\underline{U}(\xi)) \bar{V}(\xi) \\
& =c \sigma \alpha e^{\alpha \xi}-d_{N} \sigma \alpha^{2} e^{\alpha \xi}-f\left(N^{0}-\sigma e^{\alpha \xi}\right) e^{\lambda_{1} \xi} \\
& =\left[c \sigma \alpha-d_{N} \sigma \alpha^{2}-f\left(N^{0}-\sigma e^{\alpha \xi}\right) e^{\left(\lambda_{1}-\alpha\right) \xi}\right] e^{\alpha \xi} \\
& \geq\left[\left(c-d_{N} \alpha\right) \alpha \sigma-f\left(N^{0}\right)\right] e^{\alpha \xi} \\
& \geq 0 .
\end{aligned}
$$

Thus the proof is completed. 
Lemma 4. Let $\varepsilon<\alpha<\min \left\{\lambda_{1}, \lambda_{2}-\lambda_{1}\right\} / 2$. Then for $M>0$ large enough, the function $\underline{V}(\xi)$ satisfies

$$
c \underline{V^{\prime}} \leq d_{B} \underline{V}^{\prime \prime}+\kappa f(\underline{U}) \underline{V}-d \underline{V}-\gamma \underline{V^{2}},
$$

for any $\xi \neq 1 / \varepsilon \ln (1 / M)$.

Proof. It is clear that $\underline{U}(\xi)=0$ if and only if $\xi=1 / \alpha \ln \left(N^{0} / \sigma\right)$, that $\underline{V}(\xi)=0$ if and only if $\xi=1 / \varepsilon \ln (1 / M)$, and that $1 / \varepsilon \ln (1 / M)<1 / \alpha \ln \left(N^{0} / \sigma\right)$ if and only if $M>\left(\sigma / N^{0}\right)^{(\varepsilon / \alpha)}$. Assume $M>\left(\sigma / N^{0}\right)^{(\varepsilon / \alpha)}$. When $\xi>1 / \varepsilon \ln (1 / M)$, then $e^{\lambda_{1} \xi}\left(1-M e^{\varepsilon \xi}\right)<0, V(\xi)=0$, and Lemma 4 holds.

In this paragraph, assume $\xi<1 / \varepsilon \ln (1 / M)$. Then $\xi<$ $1 / \alpha \ln \left(N^{0} / \sigma\right), \underline{U}(\xi)=N^{0}-\sigma e^{\alpha \xi}>0$, and $\underline{V}(\xi)=e^{\lambda_{1} \xi}(1-$ $\left.M e^{\varepsilon \xi}\right)>0$. To prove this lemma, it is enough to show

$$
\begin{aligned}
0 \leq & e^{-\lambda_{1} \xi}\left[d_{B} \underline{V}^{\prime \prime}-c \underline{V}^{\prime}+\kappa f(\underline{U}) \underline{V}-d \underline{V}-\gamma \underline{V}^{2}\right] \\
= & d_{B} \lambda_{1}^{2}-d_{B} M\left(\lambda_{1}+\varepsilon\right)^{2} e^{\varepsilon \xi}-c \lambda_{1}+c M\left(\lambda_{1}+\varepsilon\right) e^{\varepsilon \xi} \\
& -d+d M e^{\varepsilon \xi} \\
& +\kappa\left[f\left(N^{0}\right)-f^{\prime}\left(\underline{U}^{0}\right) \sigma e^{\alpha \xi}\right]\left(1-M e^{\varepsilon \xi}\right) \\
& -\gamma e^{\lambda_{1} \xi}\left(1-M e^{\varepsilon \xi}\right)^{2} \\
= & d_{B} \lambda_{1}^{2}-c \lambda_{1}+\kappa f\left(N^{0}\right)-d \\
& \left.+M\left[-d_{B}(\lambda)_{1}+\varepsilon\right)^{2}+c\left(\lambda_{1}+\varepsilon\right)-\kappa f\left(N^{0}\right)+d\right] e^{\varepsilon \xi} \\
& -\kappa f^{\prime}\left(\underline{U}^{0}\right) \sigma e^{\alpha \xi}-\gamma e^{\lambda_{1} \xi}\left(1-M e^{\varepsilon \xi}\right)^{2}+M \kappa f^{\prime}\left(\underline{U}^{0}\right) \sigma e^{\alpha \xi} e^{\varepsilon \xi} \\
= & -M H\left(\lambda_{1}+\varepsilon\right)-\kappa f^{\prime}\left(\underline{U^{0}}\right) \sigma e^{(\alpha-\varepsilon) \xi} \\
& \left.-\gamma\left(1-M e^{\varepsilon \xi}\right)^{2} e^{\left(\lambda_{1}-\varepsilon\right) \xi}\right] e^{\varepsilon \xi} \\
& +M \kappa f^{\prime}\left(\underline{U^{0}}\right) \sigma e^{\alpha \xi} e^{\varepsilon \xi},
\end{aligned}
$$

where $\underline{U}(\xi)<\underline{U}^{0}<N^{0}$. Since $f^{\prime}\left(\underline{U}^{0}\right)>0$, we only need to show

$$
-M H\left(\lambda_{1}+\varepsilon\right) \geq \kappa f^{\prime}\left(\underline{U}^{0}\right) \sigma e^{(\alpha-\varepsilon) \xi}+\gamma\left(1-M e^{\varepsilon \xi}\right)^{2} e^{\left(\lambda_{1}-\varepsilon\right) \xi} .
$$

Since $\xi<1 / \alpha \ln \left(N^{0} / \sigma\right)<0$ by $\sigma>N^{0}$ and $0<f^{\prime}(N)<k_{1}$ for any $N \geq 0$, we have

$$
\begin{aligned}
& \kappa k_{1} \sigma>\kappa f^{\prime}\left(\underline{U}^{0}\right) \sigma e^{(\alpha-\varepsilon) \xi}, \\
& \gamma \geq \gamma\left(1-M e^{\varepsilon \xi}\right)^{2} e^{\left(\lambda_{1}-\varepsilon\right) \xi} .
\end{aligned}
$$

Since $H\left(\lambda_{1}+\varepsilon\right)<0$, inequality (17) is satisfied if

$$
M>-\frac{\kappa k_{1} \sigma+\gamma}{H\left(\lambda_{1}+\varepsilon\right)} .
$$

The proof is completed.
To apply Schauder's fixed point theorem, we will introduce a topology in $C\left(\mathbb{R}, \mathbb{R}^{2}\right)$. Let $\Lambda_{11}<0<\Lambda_{12}$ be the roots of

$$
d_{N} \Lambda^{2}-c \Lambda-\beta_{1}=0
$$

and $\Lambda_{21}<0<\Lambda_{22}$ the roots of

$$
d_{B} \Lambda^{2}-c \Lambda-\beta_{2}=0,
$$

where $\beta_{1}$ and $\beta_{2}$ are positive constants that will be determined later. Let $\mu$ be a positive constant which can be small enough. For $\Phi(\xi)=\left(\phi_{1}(\xi), \phi_{2}(\xi)\right)$, define

$$
\begin{gathered}
|\Phi(\cdot)|_{\mu}=\max \left\{\sup _{\xi \in \mathbb{R}}\left|\phi_{1}(\xi)\right| e^{-\mu|\xi|}, \sup _{\xi \in \mathbb{R}}\left|\phi_{2}(\xi)\right| e^{-\mu|\xi|}\right\}, \\
B_{\mu}\left(\mathbb{R}, \mathbb{R}^{2}\right)=\left\{\Phi(\cdot) \in C\left(\mathbb{R}, \mathbb{R}^{2}\right):|\Phi(\cdot)|_{\mu}<+\infty\right\} .
\end{gathered}
$$

We will find the traveling wave solution in the following profile set:

$$
\begin{gathered}
\Gamma=\left\{(U(\cdot), V(\cdot)) \in C\left(\mathbb{R}, \mathbb{R}^{2}\right): \underline{U}(\xi) \leq U(\xi) \leq N^{0},\right. \\
\underline{V}(\xi) \leq V(\xi) \leq \bar{V}(\xi) \text { for any } \xi \in \mathbb{R}\} .
\end{gathered}
$$

Obviously, $\Gamma$ is closed and convex in $C\left(\mathbb{R}, \mathbb{R}^{2}\right)$. Firstly, we change system (6) into the following form:

$$
\begin{aligned}
& -d_{N} U^{\prime \prime}+c U^{\prime}+\beta_{1} U=H_{1}(U, V)(\xi), \\
& -d_{B} V^{\prime \prime}+c V^{\prime}+\beta_{2} V=H_{2}(U, V)(\xi),
\end{aligned}
$$

where $\beta_{1} \geq V^{0}, \beta_{2} \geq 2 \gamma V^{0}+d=2\left[\kappa f\left(N^{0}\right)-d\right]+d$, and

$$
\begin{aligned}
& H_{1}(U, V)(\xi)=\beta_{1} U(\xi)-f(U(\xi)) V(\xi) \\
& H_{2}(U, V)(\xi)=\left[\beta_{2}-d+\kappa f(U(\xi))-\gamma V(\xi)\right] V(\xi)
\end{aligned}
$$

Furthermore, define $F=\left(F_{1}, F_{2}\right): \Gamma \rightarrow C\left(\mathbb{R}, \mathbb{R}^{2}\right)$ by

$$
\begin{aligned}
& F_{1}(U(\cdot), V(\cdot))(\xi) \\
& =\frac{1}{d_{N} \Lambda_{1}}\left[\int_{-\infty}^{\xi} e^{\Lambda_{11}(\xi-t)} H_{1}(U, V)(t) d t\right. \\
& \left.+\int_{\xi}^{+\infty} e^{\Lambda_{12}(\xi-t)} H_{1}(U, V)(t) d t\right], \\
& F_{2}(U(\cdot), V(\cdot))(\xi) \\
& =\frac{1}{d_{B} \Lambda_{2}}\left[\int_{-\infty}^{\xi} e^{\Lambda_{21}(\xi-t)} H_{2}(U, V)(t) d t\right. \\
& \left.+\int_{\xi}^{+\infty} e^{\Lambda_{22}(\xi-t)} H_{2}(U, V)(t) d t\right],
\end{aligned}
$$

where $\Lambda_{1}=\Lambda_{12}-\Lambda_{11}, \Lambda_{2}=\Lambda_{22}-\Lambda_{21}$.

Lemma 5. Consider $F(\Gamma) \subset \Gamma$. 
Proof. Suppose $(U(\cdot), V(\cdot)) \in \Gamma$; that is, $\underline{U}(\xi) \leq U(\xi) \leq$ $N^{0}, \underline{V}(\xi) \leq V(\xi) \leq \bar{V}(\xi)$ for any $\xi \in \mathbb{R}$. Then we will prove that

$$
\begin{gathered}
\underline{U}(\xi) \leq F_{1}(U(\cdot), V(\cdot))(\xi) \leq N^{0}, \\
\underline{V}(\xi) \leq F_{2}(U(\cdot), V(\cdot))(\xi) \leq \bar{V}(\xi),
\end{gathered}
$$

for any $\xi \in \mathbb{R}$.

If $\xi \geq \xi_{0} \triangleq 1 / \varepsilon \ln (1 / M)$, then $\underline{V}(\xi)=0$, which implies that $F_{2}(U(\cdot), V(\cdot))(\xi) \geq \underline{V}(\xi)$ since $\bar{U}(\xi) \geq \underline{U}(\xi) \geq 0, V(\xi) \geq$ $\underline{V}(\xi) \geq 0$. Assume $\xi<\xi_{0}$. From Lemma 4 and $\beta_{2} \geq 2 \gamma V^{0}+d$, it is clear that

$$
\begin{aligned}
-d_{B} & \underline{V}^{\prime \prime}+c \underline{V}^{\prime}+\beta_{2} \underline{V}(\xi) \\
& \leq\left[\beta_{2}-d+\kappa f(\underline{U}(\xi))-\gamma \underline{V}(\xi)\right] \underline{V}(\xi) \\
& \leq\left[\beta_{2}-d+\kappa f(U(\xi))-\gamma V(\xi)\right] V(\xi) \\
& =H_{2}(U, V)(\xi),
\end{aligned}
$$

which implies that

$$
\begin{aligned}
& F_{2}(U(\cdot), V(\cdot))(\xi) \\
& =\frac{1}{d_{B} \Lambda_{2}}\left[\int_{-\infty}^{\xi} e^{\Lambda_{21}(\xi-t)} H_{2}(U, V)(t) d t\right. \\
& \left.+\int_{\xi}^{+\infty} e^{\Lambda_{22}(\xi-t)} H_{2}(U, V)(t) d t\right] \\
& \geq \frac{1}{d_{B} \Lambda_{2}} \int_{-\infty}^{\xi} e^{\Lambda_{21}(\xi-t)}\left[-d_{B} \underline{V}^{\prime \prime}(t)+c \underline{V}^{\prime}(t)+\beta_{2} \underline{V}(t)\right] d t \\
& +\frac{1}{d_{B} \Lambda_{2}} \int_{\xi}^{\xi_{0}} e^{\Lambda_{22}(\xi-t)}\left[-d_{B} \underline{V}^{\prime \prime}(t)+c \underline{V}^{\prime}(t)+\beta_{2} \underline{V}(t)\right] d t \\
& +\frac{1}{d_{B} \Lambda_{2}} \int_{\xi_{0}}^{+\infty} e^{\Lambda_{22}(\xi-t)}\left[-d_{B} \underline{V}^{\prime \prime}(t)+c \underline{V}^{\prime}(t)+\beta_{2} \underline{V}(t)\right] d t \\
& =\underline{V}(\xi)+\frac{1}{\Lambda_{2}} e^{\Lambda_{22}\left(\xi-\xi_{0}\right)}\left[\underline{V}^{\prime}\left(\xi_{0}+0\right)-\underline{V}^{\prime}\left(\xi_{0}-0\right)\right] \\
& \geq \underline{V}(\xi),
\end{aligned}
$$

where the final inequality is due to $\underline{V}^{\prime}\left(\xi_{0}+0\right)=0$ and $\underline{V}^{\prime}\left(\xi_{0}-\right.$ $0)<0$. In conclusion, $F_{2}(U(\cdot), V(\cdot) \overline{(\xi)} \geq \underline{V}(\xi)$ for any $\bar{\xi} \in \mathbb{R}$.

Similarly, it can be proved that

$$
\begin{gathered}
\underline{U}(\xi) \leq F_{1}(U(\cdot), V(\cdot))(\xi) \leq N^{0}, \\
F_{2}(U(\cdot), V(\cdot))(\xi) \leq \bar{V}(\xi),
\end{gathered}
$$

for any $\xi \in \mathbb{R}$. The proof is completed.

Lemma 6. For $\mu$ small enough, map $F=\left(F_{1}, F_{2}\right): \Gamma \rightarrow$ $C\left(\mathbb{R}, \mathbb{R}^{2}\right)$ is continuous with respect to the norm $|\cdot|_{\mu}$ in $B_{\mu}\left(\mathbb{R}, \mathbb{R}^{2}\right)$.
Proof. Suppose $\Phi_{i}(\cdot)=\left(U_{i}(\cdot), V_{i}(\cdot)\right) \in \Gamma$, which implies

$$
0 \leq U_{i}(\xi) \leq N^{0}, \quad 0 \leq V_{i}(\xi) \leq V^{0}
$$

for any $\xi \in \mathbb{R}$, where $i=1,2$. Then we have

$$
\begin{aligned}
\left|H_{2}\left(\Phi_{1}\right)(\xi)-H_{2}\left(\Phi_{2}\right)(\xi)\right| e^{-\mu|\xi|} \\
=\mid\left(\beta_{2}-d\right)\left[V_{1}(\xi)-V_{2}(\xi)\right]-\gamma\left[V_{1}(\xi)+V_{2}(\xi)\right] \\
\quad \times\left[V_{1}(\xi)-V_{2}(\xi)\right]+\kappa f\left(U_{1}(\xi)\right)\left[V_{1}(\xi)-V_{2}(\xi)\right] \\
\quad+\kappa V_{2}(\xi)\left[f\left(U_{1}(\xi)\right)-f\left(U_{2}(\xi)\right)\right] \mid e^{-\mu|\xi|} \\
\leq\left[\beta_{2}-d+2 \gamma V^{0}+\kappa f\left(N^{0}\right)\right]\left|\Phi_{1}(\cdot)-\Phi_{2}(\cdot)\right|_{\mu} \\
\quad+\kappa V_{2}(\xi) f^{\prime}\left(U^{*}\right)\left|U_{1}(\xi)-U_{2}(\xi)\right| e^{-\mu|\xi|} \\
\leq\left[\beta_{2}-d+2 \gamma V^{0}+\kappa f\left(N^{0}\right)+\kappa V^{0} f^{\prime}(0)\right]\left|\Phi_{1}(\cdot)-\Phi_{2}(\cdot)\right|_{\mu} \\
=M_{1}\left|\Phi_{1}(\cdot)-\Phi_{2}(\cdot)\right|_{\mu},
\end{aligned}
$$

where $U^{*}$ is between $U_{1}(\xi)$ and $U_{2}(\xi)$ and

$$
M_{1}=\beta_{2}-d+2 \gamma V^{0}+\kappa f\left(N^{0}\right)+\kappa V^{0} f^{\prime}(0)>0 .
$$

Therefore,

$$
\left|F_{2}\left(\Phi_{1}(\cdot)\right)(\xi)-F_{2}\left(\Phi_{2}(\cdot)\right)(\xi)\right| e^{-\mu|\xi|}
$$

$$
\begin{aligned}
\leq \frac{e^{-\mu|\xi|}}{d_{B} \Lambda_{2}}[ & \int_{-\infty}^{\xi} e^{\Lambda_{21}(\xi-t)}\left|H_{2}\left(\Phi_{1}\right)(t)-H_{2}\left(\Phi_{2}\right)(t)\right| d t \\
& \left.\quad+\int_{\xi}^{+\infty} e^{\Lambda_{22}(\xi-t)}\left|H_{2}\left(\Phi_{1}\right)(t)-H_{2}\left(\Phi_{2}\right)(t)\right| d t\right]
\end{aligned}
$$

$$
\leq \frac{M_{1} e^{-\mu|\xi|}}{d_{B} \Lambda_{2}}\left[\int_{-\infty}^{\xi} e^{\Lambda_{21}(\xi-t)+\mu|t|} d t\right.
$$

$$
\left.+\int_{\xi}^{+\infty} e^{\Lambda_{22}(\xi-t)+\mu|t|} d t\right]\left|\Phi_{1}(\cdot)-\Phi_{2}(\cdot)\right|_{\mu}
$$


Set $\mu<\min \left\{-\Lambda_{21}, \Lambda_{22}\right\}$. If $\xi<0$, it holds that

$$
\begin{aligned}
& \left|F_{2}\left(\Phi_{1}(\cdot)\right)(\xi)-F_{2}\left(\Phi_{2}(\cdot)\right)(\xi)\right| e^{-\mu|\xi|} \\
& \leq \frac{M_{1} e^{\mu \xi}}{d_{B} \Lambda_{2}}\left[e^{\Lambda_{21} \xi} \int_{-\infty}^{\xi} e^{-\left(\Lambda_{21}+\mu\right) t} d t+e^{\Lambda_{22} \xi} \int_{\xi}^{0} e^{-\left(\Lambda_{22}+\mu\right) t} d t\right. \\
& \left.+e^{\Lambda_{22} \xi} \int_{0}^{+\infty} e^{\left(\mu-\Lambda_{22}\right) t} d t\right]\left|\Phi_{1}(\cdot)-\Phi_{2}(\cdot)\right|_{\mu} \\
& =\frac{M_{1}}{d_{B} \Lambda_{2}}\left[\frac{1}{-\Lambda_{21}-\mu}+\frac{1-e^{\left(\Lambda_{22}+\mu\right) \xi}}{\Lambda_{22}+\mu}+\frac{e^{\left(\Lambda_{22}+\mu\right) \xi}}{\Lambda_{22}-\mu}\right] \\
& \times\left|\Phi_{1}(\cdot)-\Phi_{2}(\cdot)\right|_{\mu} \\
& \leq \frac{M_{1}}{d_{B} \Lambda_{2}}\left(\frac{1}{-\Lambda_{21}-\mu}+\frac{1}{\Lambda_{22}+\mu}+\frac{1}{\Lambda_{22}-\mu}\right) \\
& \times\left|\Phi_{1}(\cdot)-\Phi_{2}(\cdot)\right|_{\mu}
\end{aligned}
$$

If $\xi \geq 0$, we have

$$
\begin{aligned}
& \left|F_{2}\left(\Phi_{1}(\cdot)\right)(\xi)-F_{2}\left(\Phi_{2}(\cdot)\right)(\xi)\right| e^{-\mu|\xi|} \\
& \leq \frac{M_{1} e^{-\mu \xi}}{d_{B} \Lambda_{2}}\left[e^{\Lambda_{21} \xi} \int_{-\infty}^{0} e^{-\left(\Lambda_{21}+\mu\right) t} d t+e^{\Lambda_{21} \xi} \int_{0}^{\xi} e^{\left(\mu-\Lambda_{21}\right) t} d t\right. \\
& \left.+e^{\Lambda_{22} \xi} \int_{\xi}^{+\infty} e^{\left(\mu-\Lambda_{22}\right) t} d t\right]\left|\Phi_{1}(\cdot)-\Phi_{2}(\cdot)\right|_{\mu} \\
& =\frac{M_{1}}{d_{B} \Lambda_{2}}\left[\frac{e^{\left(\Lambda_{21}-\mu\right) \xi}}{-\Lambda_{21}-\mu}+\frac{1-e^{\left(\Lambda_{21}-\mu\right) \xi}}{\mu-\Lambda_{21}}+\frac{1}{\Lambda_{22}-\mu}\right] \\
& \times\left|\Phi_{1}(\cdot)-\Phi_{2}(\cdot)\right|_{\mu} \\
& \leq \frac{M_{1}}{d_{B} \Lambda_{2}}\left(\frac{1}{-\Lambda_{21}-\mu}+\frac{1}{\mu-\Lambda_{21}}+\frac{1}{\Lambda_{22}-\mu}\right) \\
& \times\left|\Phi_{1}(\cdot)-\Phi_{2}(\cdot)\right|_{\mu} \cdot
\end{aligned}
$$

Consequently, we conclude that

$$
\left|F_{2}\left(\Phi_{1}(\cdot)\right)(\cdot)-F_{2}\left(\Phi_{2}(\cdot)\right)(\cdot)\right|_{\mu} \leq M_{2}\left|\Phi_{1}(\cdot)-\Phi_{2}(\cdot)\right|_{\mu}
$$

where

$$
\begin{aligned}
M_{2}=\frac{M_{1}}{d_{B} \Lambda_{2}} \max \{ & \frac{1}{-\Lambda_{21}-\mu}+\frac{1}{\Lambda_{22}+\mu}+\frac{1}{\Lambda_{22}-\mu}, \\
& \left.\frac{1}{-\Lambda_{21}-\mu}+\frac{1}{\mu-\Lambda_{21}}+\frac{1}{\Lambda_{22}-\mu}\right\} .
\end{aligned}
$$

Thus $F_{2}: \Gamma \rightarrow C(\mathbb{R}, \mathbb{R})$ is continuous with respect to the norm $|\cdot|_{\mu}$ in $B_{\mu}(\mathbb{R}, \mathbb{R})$. Similarly, it can be proved that $F_{1}$ : $\Gamma \rightarrow C(\mathbb{R}, \mathbb{R})$ is also continuous with respect to the norm $|\cdot|_{\mu}$ in $B_{\mu}(\mathbb{R}, \mathbb{R})$. The proof is completed.
Lemma 7. Map $F=\left(F_{1}, F_{2}\right): \Gamma \rightarrow \Gamma$ is compact with respect to the norm $|\cdot|_{\mu}$ in $B_{\mu}\left(\mathbb{R}, \mathbb{R}^{2}\right)$.

Proof. Assume $\Phi(\cdot)=(U(\cdot), V(\cdot)) \in \Gamma$. Then we have

$$
\left|H_{2}(\Phi)(\xi)\right|=\left|\left[\beta_{2}-d+\kappa f(U(\xi))-\gamma V(\xi)\right] V(\xi)\right| \leq M_{3},
$$

where

$$
M_{3}=\left(\beta_{2}+d+\frac{\kappa k_{1}}{k_{2}}+\gamma V^{0}\right) V^{0} .
$$

Then

$$
\begin{aligned}
& \left|\frac{d}{d \xi} F_{2}(\Phi(\cdot))(\xi)\right| \\
& =\frac{1}{d_{B} \Lambda_{2}} \mid \Lambda_{21} \int_{-\infty}^{\xi} e^{\Lambda_{21}(\xi-t)} H_{2}(\Phi)(t) d t \\
& +\Lambda_{22} \int_{\xi}^{+\infty} e^{\Lambda_{22}(\xi-t)} H_{2}(\Phi)(t) d t \\
& \leq \frac{M_{3}}{d_{B} \Lambda_{2}}\left[\left|\Lambda_{21}\right| \int_{-\infty}^{\xi} e^{\Lambda_{21}(\xi-t)} d t+\Lambda_{22} \int_{\xi}^{+\infty} e^{\Lambda_{22}(\xi-t)} d t\right] \\
& =\frac{2 M_{3}}{d_{B} \Lambda_{2}} \text {, }
\end{aligned}
$$

which implies

$$
\left|\frac{d}{d \xi} F_{2}(\Phi(\cdot))(\cdot)\right|_{\mu}<\frac{2 M_{3}}{d_{B} \Lambda_{2}} .
$$

Consequently, $\left|(d / d \xi) F_{2}(\Phi(\cdot))(\cdot)\right|_{\mu}$ is bounded. Similarly, $\left|(d / d \xi) F_{1}(\Phi(\cdot))(\cdot)\right|_{\mu}$ is also bounded, which shows that $F(\Gamma)$ is uniformly bounded and equicontinuous with respect to the norm $|\cdot|_{\mu}$.

Furthermore, for any positive integer $n$, we define

$$
F^{n}(\Phi(\cdot))(\xi)= \begin{cases}F(\Phi(\cdot))(\xi), & \xi \in[-n, n], \\ F(\Phi(\cdot))(-n), & \xi \in(-\infty,-n], \\ F(\Phi(\cdot))(n), & \xi \in[n,+\infty) .\end{cases}
$$

Obviously, for fixed $n, F^{n}(\Gamma)$ is uniformly bounded and equicontinuous with respect to the norm $|\cdot|_{\mu}$ in $B_{\mu}\left(\mathbb{R}, \mathbb{R}^{2}\right)$, implying that $F^{n}: \Gamma \rightarrow \Gamma$ is a compact operator. Since

$$
\begin{aligned}
& \left|F_{2}(\Phi(\cdot))(\xi)\right| \\
& \quad \leq \frac{M_{3}}{d_{B} \Lambda_{2}}\left[\int_{-\infty}^{\xi} e^{\Lambda_{21}(\xi-t)} d t+\int_{\xi}^{+\infty} e^{\Lambda_{22}(\xi-t)} d t\right] \\
& \quad=\frac{M_{3}}{d_{B}\left|\Lambda_{21}\right| \Lambda_{22}},
\end{aligned}
$$


we have

$$
\begin{aligned}
& \left|F_{2}^{n}(\Phi(\cdot))(\cdot)-F_{2}(\Phi(\cdot))(\cdot)\right|_{\mu} \\
& =\sup _{\xi \in \mathbb{R}}\left|F_{2}^{n}(\Phi(\cdot))(\xi)-F_{2}(\Phi(\cdot))(\xi)\right| e^{-\mu|\xi|} \\
& =\sup _{\xi \in(-\infty,-n] \cup[n,+\infty)}\left|F_{2}^{n}(\Phi(\cdot))(\xi)-F_{2}(\Phi(\cdot))(\xi)\right| e^{-\mu|\xi|} \\
& \leq \frac{2 M_{3}}{d_{B}\left|\Lambda_{21}\right| \Lambda_{22}} e^{-\mu n} \longrightarrow 0, \text { as } n \longrightarrow+\infty .
\end{aligned}
$$

Similarly, we can prove that

$$
\left|F_{1}^{n}(\Phi(\cdot))(\cdot)-F_{1}(\Phi(\cdot))(\cdot)\right|_{\mu} \longrightarrow 0,
$$

when $n \rightarrow+\infty$. Thus, $\left|F^{n}(\Phi(\cdot))(\cdot)-F(\Phi(\cdot))(\cdot)\right|_{\mu} \rightarrow 0$ when $n \rightarrow+\infty$. By Proposition 2.1 in Zeidler [30] we see that $F^{n}$ converges to $F$ in $\Gamma$ with respect to the norm $|\cdot|_{\mu}$. Consequently, $F: \Gamma \rightarrow \Gamma$ is compact with respect to the norm $|\cdot|_{\mu}$. The proof is completed.

Lemma 8. Let $c>c^{*}$; then (6) has a solution $(U(\xi), V(\xi))$ satisfying (3):

$$
\begin{array}{r}
\int_{-\infty}^{+\infty}\left[d V(\eta)+\gamma V^{2}(\eta)\right] d \eta=\kappa c\left(N^{0}-N^{1}\right), \\
0 \leq V(\xi) \leq \kappa\left(N^{0}-N^{1}\right),
\end{array}
$$

for any $\xi \in \mathbb{R}$.

Proof. Combination of Schauder's fixed point theorem, Lemmas 5, 6, and 7 shows that there exists a nonnegative traveling wave solution $\left(U_{c}(\cdot), V_{c}(\cdot)\right) \in \Gamma$ such that $\left(U_{c}(\xi), V_{c}(\xi)\right) \rightarrow$ $\left(N^{0}, 0\right)$ when $\xi \rightarrow-\infty$. Since $\left(U_{c}(\cdot), V_{c}(\cdot)\right)$ is the fixed point of $F$, L'Hospital principal shows that $U^{\prime}(-\infty)=0, V^{\prime}(-\infty)=$ 0 . Then from $(6)$ we have that $U^{\prime \prime}(-\infty)=0, V^{\prime \prime}(-\infty)=0$. Since $\left(U_{c}(\xi), V_{c}(\xi)\right)$ is the solution of $(6)$, thus

$$
\begin{aligned}
& c U_{c}^{\prime}=d_{N} U_{c}^{\prime \prime}-f\left(U_{c}\right) V_{c}, \\
& c V_{c}^{\prime}=d_{B} V_{c}^{\prime \prime}+\kappa f\left(U_{c}\right) V_{c}-d V_{c}-\gamma V_{c}^{2} .
\end{aligned}
$$

The first equation of (48) can be changed into

$$
\frac{c}{d_{N}} U_{c}^{\prime}-U_{c}^{\prime \prime}=-\frac{1}{d_{N}} f\left(U_{c}\right) V_{c} .
$$

Multiplying this equation by $e^{-c / d_{N} \xi}$ yields

$$
-\left[e^{-c / d_{N} \xi} U_{c}^{\prime}(\xi)\right]^{\prime}=-\frac{1}{d_{N}} f\left(U_{c}\right) V_{c} e^{-c / d_{N} \xi} .
$$

From the proof of Lemma 7 , we have $U_{c}^{\prime}(\xi)=$ $F_{1}^{\prime}\left(U_{c}(\cdot), V_{c}(\cdot)\right)(\xi)$ is bounded in $\mathbb{R}$. Then integrating above equality from $\xi$ to $+\infty$, we have

$$
U_{c}^{\prime}(\xi)=-\frac{1}{d_{N}} e^{c / d_{N} \xi} \int_{\xi}^{+\infty} f\left(U_{c}(\eta)\right) V_{c}(\eta) e^{-c / d_{N} \eta} d \eta \leq 0
$$

which implies that $U_{c}(\xi)$ is nonincreasing in $\mathbb{R}$ and has limit $N^{1}$ as $\xi \rightarrow+\infty$. By the definition of $\underline{U}(\xi)$ and $\underline{V}(\xi)$ there is a $\xi_{0}<0$ such that $\underline{U}(\xi)>0$ and $\underline{V}(\xi)>0$ when $\xi<\xi_{0}$. Therefore, if $\xi<\xi_{0}$, we have that $U_{c}^{\top}(\xi)<0$ which implies that $N^{0}>N^{1} \geq 0$.

Integrating the first equation of (48) from $-\infty$ to $\xi$ gives

$$
\int_{-\infty}^{\xi} f\left(U_{c}(\eta)\right) V_{c}(\eta) d \eta=d_{N} U_{c}^{\prime}(\xi)-c\left[U_{c}(\xi)-N^{0}\right],
$$

which implies that $\int_{-\infty}^{+\infty} f\left(U_{c}(\eta)\right) V_{c}(\eta) d \eta<+\infty$. Integrating the second equation of (48) from $-\infty$ to $\xi$ gives

$$
\begin{aligned}
c V_{c}(\xi)= & d_{B} V_{c}^{\prime}(\xi)+\int_{-\infty}^{\xi} \kappa f\left(U_{c}(\eta)\right) V_{c}(\eta) d \eta \\
& -d \int_{-\infty}^{\xi} V_{c}(\eta) d \eta-\gamma \int_{-\infty}^{\xi} V_{c}^{2}(\eta) d \eta .
\end{aligned}
$$

Thus $\int_{-\infty}^{+\infty} V_{c}(\eta) d \eta<+\infty$ and $\lim _{\xi \rightarrow+\infty} V_{c}(\xi)=0$ since $V_{c}^{\prime}(\xi)$ is bounded in $\mathbb{R}$. By (51) and L'Hospital principal, it follows $U_{c}^{\prime}(+\infty)=0$. Then using (52) and (53) shows that

$$
\int_{-\infty}^{+\infty}\left[d V_{c}(\eta)+\gamma V_{c}^{2}(\eta)\right] d \eta=\kappa c\left(N^{0}-N^{1}\right)
$$

Next, we prove that $0 \leq V_{c}(\xi) \leq d\left(N^{0}-N^{1}\right) /\left(d-\alpha_{2}\right)$. Let

$$
\begin{aligned}
R(\xi)= & \frac{1}{c} \int_{-\infty}^{\xi}\left[d V_{c}(\eta)+\gamma V_{c}^{2}(\eta)\right] d \eta \\
& +\frac{1}{c} \int_{\xi}^{+\infty} e^{c(\xi-\eta) / d_{B}}\left[d V_{c}(\eta)+\gamma V_{c}^{2}(\eta)\right] d \eta
\end{aligned}
$$

It is clear that $R(-\infty)=0$ and $R(+\infty)=\kappa\left(N^{0}-N^{1}\right)$. Define $S(\xi)=V_{c}(\xi)+R(\xi)$. Calculations show that

$$
c S^{\prime}(\xi)-d_{B} S^{\prime \prime}(\xi)=\kappa f\left(U_{c}(\xi)\right) V_{c}(\xi) .
$$

Multiplying this equality by $e^{-c \xi / d_{B}}$ and then integrating from $\xi$ to $+\infty$ show that

$$
S^{\prime}(\xi)=\frac{\kappa}{d_{B}} \int_{\xi}^{+\infty} e^{c(\xi-\zeta) / d_{B}}\left[f\left(U_{c}(\zeta)\right) V_{c}(\zeta) d \eta\right] d \zeta \geq 0
$$

for any $\xi \in \mathbb{R}$. Consequently, $S(\xi)$ is nondecreasing in $\mathbb{R}$. Since

$$
S(+\infty)=R(+\infty)=\kappa\left(N^{0}-N^{1}\right)
$$

we have that $0 \leq V_{c}(\xi) \leq \kappa\left(N^{0}-N^{1}\right)$ for any $\xi \in \mathbb{R}$. The proof is completed.

Proof of Theorem 1. Firstly, we consider the case $c>c^{*}$. Let $\left\{\varepsilon_{n}\right\}$ be a sequence such that $0<\varepsilon_{i+1}<\varepsilon_{i}<1$ and $\varepsilon_{n} \rightarrow 0$. By Lemma 8 , there exists a traveling wave solution 
$\Phi_{n}(\xi)=\left(U_{n}(\xi), V_{n}(\xi)\right)$ of system (6) for $\gamma=\varepsilon_{n}$ satisfying the conclusion of Theorem 1. From (51), we have

$$
\begin{aligned}
\left|U_{n}^{\prime}(\xi)\right| & =\frac{1}{d_{N}} e^{c / d_{N} \xi} \int_{\xi}^{+\infty} f\left(U_{n}(\eta)\right) V_{n}(\eta) e^{-c / d_{N} \eta} d \eta \leq 0 \\
& \leq \frac{f\left(N^{0}\right) \kappa\left(N^{0}-N^{1}\right)}{d_{N}} e^{c / d_{N} \xi} \int_{\xi}^{+\infty} e^{-c / d_{N} \eta} d \eta \\
& =\frac{f\left(N^{0}\right) \kappa\left(N^{0}-N^{1}\right)}{c} .
\end{aligned}
$$

Similarly, it can be shown that $\left|V_{n}^{\prime}(\xi)\right| \leq M_{0}$, where $M_{0}$ is independent of $\varepsilon_{n}$ due to $\varepsilon_{n}<1$. By (6), there is a positive constant $M_{4}$ independent of $\varepsilon_{n}$ such that $\left|U_{n}^{\prime \prime}(\xi)\right|,\left|V_{n}^{\prime \prime}(\xi)\right|$, $\left|U_{n}^{\prime \prime \prime}(\xi)\right|$, and $\left|V_{n}^{\prime \prime \prime}(\xi)\right|$ are bounded in $\xi \in \mathbb{R}$ by $M_{4}$.

Therefore, $\left\{\Phi_{n}(\xi)\right\},\left\{\Phi_{n}^{\prime}(\xi)\right\},\left\{\Phi_{n}^{\prime \prime}(\xi)\right\}$ are equicontinuous and uniformly bounded in $\mathbb{R}$. Then Arzela-Ascoli's theorem implies that there exists a subsequence $\left\{\varepsilon_{n_{k}}\right\}$ such that

$$
\begin{gathered}
\Phi_{n_{k}}(\xi) \longrightarrow \\
\Phi^{\prime \prime}(\xi), \quad \Phi_{n_{k}}^{\prime}(\xi) \longrightarrow \Psi^{\prime}(\xi),
\end{gathered}
$$

uniformly in any bounded closed interval when $k \rightarrow \infty$ and pointwise on $\mathbb{R}$, where $\Psi(\xi)=\left(\psi_{1}(\xi), \psi_{2}(\xi)\right)$. Since $\Phi_{n_{k}}(\xi)$ is the solution of (6) and $\varepsilon_{n} \rightarrow 0$, we get

$$
\begin{aligned}
& c \psi_{1}^{\prime}(\xi)=d_{N} \psi_{1}^{\prime \prime}(\xi)-f\left(\psi_{1}(\xi)\right) \psi_{2}(\xi), \\
& c \psi_{2}^{\prime}(\xi)=d_{B} \psi_{2}^{\prime \prime}(\xi)+\kappa f\left(\psi_{1}(\xi)\right) \psi_{2}(\xi)-d \psi_{2}(\xi) .
\end{aligned}
$$

That is, $\Psi(\xi)$ is a solution of (5) satisfying (3):

$$
\begin{array}{r}
\int_{-\infty}^{+\infty} \psi_{2}(\eta) d \eta=\frac{\kappa c}{d}\left(N^{0}-N^{1}\right), \\
0 \leq \psi_{2}(\xi) \leq \kappa\left(N^{0}-N^{1}\right) .
\end{array}
$$

To complete the proof of case $c>c^{*}$, we need to prove $f\left(N^{1}\right)<d / \kappa$. Integrating the second equation of system (5) from $-\infty$ to $+\infty$ and noting that $U(\xi)$ is decreasing from $N^{0}$ to $N^{1}$, we have

$$
\begin{aligned}
& d \int_{-\infty}^{+\infty} V(\xi) d \xi \\
& \quad=\kappa \int_{-\infty}^{+\infty} f(U(\xi)) V(\xi) d \xi>\kappa f\left(N^{1}\right) \int_{-\infty}^{+\infty} V(\xi) d \xi
\end{aligned}
$$

which implies $f\left(N^{1}\right)<d / \kappa$.

To prove case $c=c^{*}$, let the parameter $c=c_{n}$ in system (5), $c^{*}<c_{n}<c^{*}+1$, and $c_{n} \rightarrow c^{*}$. Similar to above proof about case $c>c^{*}$, we can finish the proof.

\section{Nonexistence of Traveling Wave Solution}

In this section, we give the conditions on which system (1) has no traveling wave solutions.
Theorem 9. (I) Assume $f\left(N^{0}\right)>d / \kappa$. Then for any $0<$ $c<c^{*}$, system (1) has no nonnegative traveling wave solutions $(U(x+c t), V(x+c t))$ satisfying boundary condition (3).

(II) Suppose $f\left(N^{0}\right) \leq d / \kappa$. Then for any $c>0$, system (1) has no traveling wave solutions $(U(x+c t), V(x+c t))$ satisfying boundary condition (3).

Proof of Theorem 9(I). Suppose (I) fails. That is, system (5) has a nonnegative nontrivial traveling wave solution $(U(\xi), V(\xi))$ satisfying boundary condition (3). Since $U(-\infty)=N^{0}$ and $f\left(N^{0}\right)>d / \kappa$, there exists a $\xi_{0}<0$ such that $f(U(\xi)) \geq\left[f\left(N^{0}\right)+d / \kappa\right] / 2$ for any $\xi<\xi_{0}$. Thus, we get

$$
\begin{aligned}
c V^{\prime}(\xi) & =d_{B} V^{\prime \prime}(\xi)+\kappa f(U(\xi)) V(\xi)-d V(\xi) \\
& \geq d_{B} V^{\prime \prime}(\xi)+\frac{\kappa f\left(N^{0}\right)+d}{2} V(\xi)-d V(\xi) \\
& =d_{B} V^{\prime \prime}(\xi)+\frac{\kappa f\left(N^{0}\right)-d}{2} V(\xi)
\end{aligned}
$$

for any $\xi \leq \xi_{0}$. That is,

$$
\frac{\kappa f\left(N^{0}\right)-d}{2} V(\xi) \leq c V^{\prime}(\xi)-d_{B} V^{\prime \prime}(\xi),
$$

for any $\xi<\xi_{0}$. Now we show $V^{\prime}(-\infty)=0$. Denote $W(\xi) \triangleq$ $V^{\prime}(\xi)$. From the second equation of (5), we have

$$
d_{B} W^{\prime}(\xi)=c W(\xi)+G(\xi)
$$

where $G(\xi)=d V(\xi)-\kappa f(U(\xi)) V(\xi)$. Since $(U(\xi), V(\xi))$ satisfies boundary condition (3), it follows $G(-\infty)=0$. If $W(-\infty) \neq 0$, then $W(-\infty)=+\infty$ or $W(-\infty)=-\infty$, which imply $V(-\infty)=-\infty$ or $V(-\infty)=+\infty$ contradicting $V(-\infty)=0$.

Defining $J(\xi)=\int_{-\infty}^{\xi} V(\eta) d \eta$ and integrating (65) from $-\infty$ to $\xi$, we have that

$$
\frac{\kappa f\left(N^{0}\right)-d}{2} J(\xi) \leq c V(\xi)-d_{B} V^{\prime}(\xi) .
$$

Integrating (67) from $-\infty$ to $\xi$ with $\xi \leq \xi_{0}$ yields

$$
\frac{\kappa f\left(N^{0}\right)-d}{2} \int_{-\infty}^{\xi} J(\eta) d \eta+d_{B} V(\xi) \leq c J(\xi) .
$$

Therefore, we get

$$
\frac{\kappa f\left(N^{0}\right)-d}{2} \int_{-\infty}^{0} J(\xi+\eta) d \eta \leq c J(\xi),
$$

for any $\xi \leq \xi_{0}$. Since $J(\eta)$ is increasing in $\mathbb{R}$, it is clear that

$$
\frac{\kappa f\left(N^{0}\right)-d}{2} \eta J(\xi-\eta) \leq c J(\xi),
$$

for any $\xi \leq \xi_{0}$ and $\eta>0$. Therefore, there is $\eta_{0}>0$ large enough such that

$$
J\left(\xi-\eta_{0}\right) \leq \frac{1}{2} J(\xi)
$$


for any $\xi \leq \xi_{0}$. Let $p(\xi)=J(\xi) e^{-\mu_{0} \xi}$ and $\mu_{0}=\left(1 / \eta_{0}\right) \ln 2$. We get that

$$
p\left(\xi-\eta_{0}\right)=J\left(\xi-\eta_{0}\right) e^{-\mu_{0}\left(\xi-\eta_{0}\right)} \leq \frac{1}{2} J(\xi) e^{-\mu_{0}\left(\xi-\eta_{0}\right)}=p(\xi),
$$

for any $\xi \leq \xi_{0}$. Since $J(\xi)$ is bounded in $\mathbb{R}$, thus $p(\xi) \rightarrow 0$ as $\xi \rightarrow+\infty$, which implies that there exists $p_{0}>0$ such that $p(\xi) \leq p_{0}$ for any $\xi \in \mathbb{R}$. Hence, we have that

$$
J(\xi) \leq p_{0} e^{\mu_{0} \xi}
$$

for $\xi \in \mathbb{R}$ and that there exists $q_{0}>0$ such that $\int_{-\infty}^{\xi} J(\eta) d \eta \leq$ $q_{0} e^{\mu_{0} \xi}$. In addition, inequalities (65)-(68) imply that

$$
\begin{gathered}
\sup _{\xi \in \mathbb{R}}\left\{V(\xi) e^{-\mu_{0} \xi}\right\}<+\infty, \\
\sup _{\xi \in \mathbb{R}}\left\{\left|V^{\prime}(\xi)\right| e^{-\mu_{0} \xi}\right\}<+\infty, \\
\sup _{\xi \in \mathbb{R}}\left\{\left|V^{\prime \prime}(\xi)\right| e^{-\mu_{0} \xi}\right\}<+\infty .
\end{gathered}
$$

To complete the proof, we define negative one-sided Laplace transform as follows:

$$
\mathscr{V}(\lambda)=\mathcal{N}[V(\cdot)](\lambda):=\int_{-\infty}^{0} e^{-\lambda \xi} V(\xi) d \xi,
$$

for $\lambda \geq 0$. Obviously $\mathscr{V}(\lambda)$ is increasing in $\left[0, \lambda^{*}\right)$ such that $\lambda^{*}<+\infty$ satisfying $\lim _{\lambda \rightarrow \lambda^{*-}} \mathscr{V}(\lambda)=+\infty$ or $\lambda^{*}=+\infty$. Since $\sup _{\xi \in \mathbb{R}}\left\{V(\xi) e^{-\mu_{0} \xi}\right\}<+\infty$, we have $\lambda^{*} \geq \mu_{0}$. Trivial calculations show that $\mathcal{N}[\cdot]$ satisfies

$$
\begin{aligned}
& \mathcal{N}\left[V^{\prime}(\cdot)\right](\lambda)=\lambda \mathscr{V}(\lambda)+V(0), \\
& \mathcal{N}\left[V^{\prime \prime}(\cdot)\right](\lambda)=\lambda^{2} \mathscr{V}(\lambda)+\lambda V(0)+V^{\prime}(0) .
\end{aligned}
$$

The second equation of (5) can be rewritten as

$$
L[V(\cdot)](\xi)=\kappa\left[f\left(N^{0}\right)-f(U(\xi))\right] V(\xi),
$$

where

$$
L[V(\cdot)](\xi)=d_{B} V^{\prime \prime}(\xi)-c V^{\prime}(\xi)+\left[\kappa f\left(N^{0}\right)-d\right] V(\xi) .
$$

Define $\rho=\min \{H(\lambda): \lambda \geq 0\}$. Noticing $0<c<c^{*}$ yields $\rho>0$. Since (5) is autonomous, then for any $a \in$ $\mathbb{R},(U(\xi-a), V(\xi-a))$ is also a solution of (5) satisfying boundary condition (3) and $U(\xi-a) \rightarrow N^{0}$ as $a \rightarrow+\infty$. Hence, without losing generality we can assume

$$
\kappa\left[f\left(N^{0}\right)-f(U(\xi))\right]<\frac{\rho}{2},
$$

for all $\xi \leq 0$. That is,

$$
L[V(\cdot)](\xi) \leq \frac{\rho}{2} V(\xi) .
$$

Applying the operator $\mathcal{N}[\cdot]$ to this inequality and using the properties of $\mathcal{N}[\cdot]$ concluded above yield that

$$
\frac{\rho}{2} \mathscr{V}(\lambda) \geq \mathcal{N}[L[V(\cdot)](\cdot)](\lambda) \geq H(\lambda) \mathscr{V}(\lambda)+q(\lambda),
$$

where $H(\lambda)$ is the characteristic function of (7) and

$$
q(\lambda)=d_{B} V^{\prime}(0)+\left(d_{B} \lambda-c\right) V(0) .
$$

Consequently, we have

$$
\mathscr{H}(\lambda):=\left[H(\lambda)-\frac{\rho}{2}\right] \mathscr{V}(\lambda)+q(\lambda) \leq 0
$$

If $\lambda^{*}<+\infty$, then $\lim _{\lambda \rightarrow \lambda^{*-}} \mathscr{V}(\lambda)=+\infty$ and, therefore, $\lim _{\lambda \rightarrow \lambda^{*-}} \mathscr{H}(\lambda)=+\infty$, which is a contradiction. If $\lambda^{*}=+\infty$, we have that $\lim _{\lambda \rightarrow+\infty} \mathscr{H}(\lambda)=+\infty$ by the monotonicity of $\mathscr{V}(\lambda)$ and the definitions of $H(\lambda)$ and $q(\lambda)$, which is still a contradiction. The proof of Theorem 9(I) is completed.

Proof of Theorem 9(II). Suppose $(U(\xi), V(\xi))$ is a nontrivial solution of system (5) satisfying boundary condition (3). Similar to the arguments about (66), it is easy to show that $V^{\prime}( \pm \infty)=0$. Then integrating the second equation of (5) from $-\infty$ to $+\infty$ yields

$$
\begin{aligned}
& d \int_{-\infty}^{+\infty} V(\xi) d \xi \\
& \quad=\kappa \int_{-\infty}^{+\infty} f(U(\xi)) V(\xi) d \xi<\kappa f\left(N^{0}\right) \int_{-\infty}^{+\infty} V(\xi) d \xi \\
& \quad \leq d \int_{-\infty}^{+\infty} V(\xi) d \xi
\end{aligned}
$$

which is a contradiction. The proof is completed.

\section{Conflict of Interests}

The authors declare that there is no conflict of interests regarding the publication of this paper.

\section{Acknowledgment}

The authors were supported by the Fundamental Research Funds for the Central Universities (XDJK2012C042 and SWU113048).

\section{References}

[1] A. Aotani, M. Mimura, and T. Mollee, "A model aided understanding of spot pattern formation in chemotactic E. coli colonies," Japan Journal of Industrial and Applied Mathematics, vol. 27, no. 1, pp. 5-22, 2010.

[2] T. Banitz, K. Johst, L. Y. Wick, I. Fetzer, H. Harms, and K. Frank, "The relevance of conditional dispersal for bacterial colony growth and biodegradation," Microbial Ecology, vol. 63, no. 2, pp. 339-347, 2012.

[3] J. A. Bonachela, C. D. Nadell, J. B. Xavier, and S. A. Levin, "Universality in bacterial colonies," Journal of Statistical Physics, vol. 144, no. 2, pp. 303-315, 2011. 
[4] J. Lega and T. Passot, "Hydrodynamics of bacterial colonies," Nonlinearity, vol. 20, no. 1, pp. C1-C16, 2007.

[5] A. Marrocco, H. Henry, I. B. Holland, M. Plapp, S. J. Séror, and B. Perthame, "Models of self-organizing bacterial communities and comparisons with experimental observations," Mathematical Modelling of Natural Phenomena, vol. 5, no. 1, pp. 148-162, 2010.

[6] M. Mimura, H. Sakaguchi, and M. Matsushita, "Reactiondiffusion modelling of bacterial colony patterns," Physica A, vol. 282, no. 1, pp. 283-303, 2000.

[7] M. K. Roy, P. Banerjee, T. K. Sengupta, and S. Dattagupta, "Glucose induced fractal colony pattern of Bacillus thuringiensis," Journal of Theoretical Biology, vol. 265, no. 3, pp. 389-395, 2010.

[8] Y. Yamazaki, T. Ikeda, H. Shimada et al., "Periodic growth of bacterial colonies," Physica D, vol. 205, no. 1-4, pp. 136-153, 2005.

[9] O. A. Croze, G. P. Ferguson, M. E. Cates, and W. C. K. Poon, "Migration of chemotactic bacteria in soft agar: role of gel concentration," Biophysical Journal, vol. 101, no. 3, pp. 525-534, 2011.

[10] B. Grammaticos, M. Badoual, and M. Aubert, "An (almost) solvable model for bacterial pattern formation," Physica D, vol. 234, no. 2, pp. 90-97, 2007.

[11] J. F. Leyva, C. Málaga, and R. G. Plaza, “The effects of nutrient chemotaxis on bacterial aggregation patterns with non-linear degenerate cross diffusion," Physica A, vol. 392, no. 22, pp. 5644$5662,2013$.

[12] A. F. Miguel, "Constructal pattern formation in stony corals, bacterial colonies and plant roots under different hydrodynamics conditions," Journal of Theoretical Biology, vol. 242, no. 4, pp. 954-961, 2006.

[13] A. Nishiyama, T. Tokihiro, M. Badoual, and B. Grammaticos, "Modelling the morphology of migrating bacterial colonies," Physica D, vol. 239, no. 16, pp. 1573-1580, 2010.

[14] A. M. A. El-Sayed, S. Z. Rida, and A. A. M. Arafa, "On the solutions of the generalized reaction-diffusion model for bacterial colony," Acta Applicandae Mathematicae, vol. 110, no. 3, pp. 1501-1511, 2010.

[15] M. Torrisi and R. Tracinà, "Exact solutions of a reactiondiffusion systems for Proteus mirabilis bacterial colonies," Nonlinear Analysis: Real World Applications, vol. 12, no. 3, pp. 1865-1874, 2011.

[16] L. Zhang, "Positive steady states of an elliptic system arising from biomathematics," Nonlinear Analysis: Real World Applications, vol. 6, no. 1, pp. 83-110, 2005.

[17] P. Feng and Z. Zhou, "Finite traveling wave solutions in a degenerate cross-diffusion model for bacterial colony," Communications on Pure and Applied Analysis, vol. 6, no. 4, pp. 11451165, 2007.

[18] M. B. A. Mansour, "Traveling wave solutions of a reactiondiffusion model for bacterial growth," Physica A, vol. 383, no. 2, pp. 466-472, 2007.

[19] M. B. A. Mansour, "Traveling wave solutions of a nonlinear reaction-diffusion-chemotaxis model for bacterial pattern formation," Applied Mathematical Modelling, vol. 32, no. 2, pp. $240-$ 247, 2008.

[20] M. B. A. Mansour, "Analysis of propagating fronts in a nonlinear diffusion model with chemotaxis," Wave Motion, vol. 50, no. 1, pp. 11-17, 2013.

[21] J. Müller and W. van Saarloos, "Morphological instability and dynamics of fronts in bacterial growth models with nonlinear diffusion," Physical Review E, vol. 65, no. 6, Article ID 061111, 2002.

[22] R. A. Satnoianu, P. K. Maini, F. S. Garduno, and J. P. Armitage, "Travelling waves in a nonlinear degenerate diffusion model for bacterial pattern formation," Discrete and Continuous Dynamical Systems B, vol. 1, no. 3, pp. 339-362, 2001.

[23] S. Thanarajah and H. Wang, "Competition of motile and immotile bacterial strains in a petri dish," Mathematical Biosciences and Engineering, vol. 10, no. 2, pp. 399-424, 2013.

[24] J. Y. Wakano, A. Komoto, and Y. Yamaguchi, "Phase transition of traveling waves in bacterial colony pattern," Physical Review E, vol. 69, no. 5, Article ID 051904, 9 pages, 2004.

[25] O. Diekmann, "Thresholds and travelling waves for the geographical spread of infection," Journal of Mathematical Biology, vol. 6, no. 2, pp. 109-130, 1978.

[26] J. Carr and A. Chmaj, "Uniqueness of travelling waves for nonlocal monostable equations," Proceedings of the American Mathematical Society, vol. 132, no. 8, pp. 2433-2439, 2004.

[27] Z.-C. Wang, W.-T. Li, and S. Ruan, "Traveling fronts in monostable equations with nonlocal delayed effects," Journal of Dynamics and Differential Equations, vol. 20, no. 3, pp. 573-607, 2008.

[28] Z.-C. Wang, W.-T. Li, and S. Ruan, "Entire solutions in bistable reaction-diffusion equations with nonlocal delayed nonlinearity," Transactions of the American Mathematical Society, vol. 361, no. 4, pp. 2047-2084, 2009.

[29] Z.-C. Wang and J. Wu, "Travelling waves of a diffusive KermackMcKendrick epidemic model with non-local delayed transmission," Proceedings of The Royal Society of London A, vol. 466, no. 2113, pp. 237-261, 2010.

[30] E. Zeidler, Nonlinear Functional Analysis and Its applications I, Springer, New York, NY, USA, 1986. 


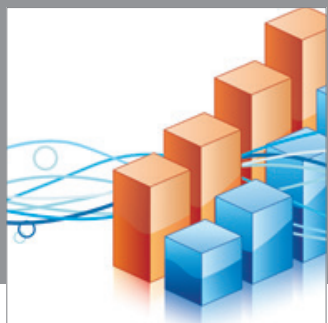

Advances in

Operations Research

mansans

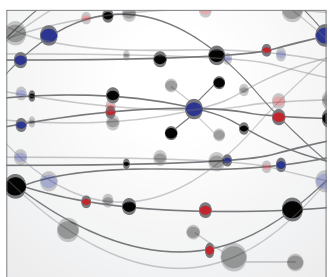

The Scientific World Journal
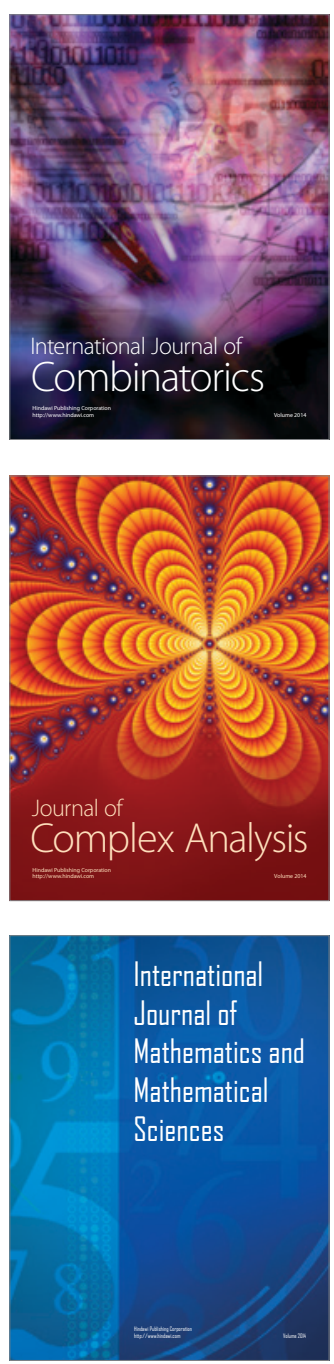
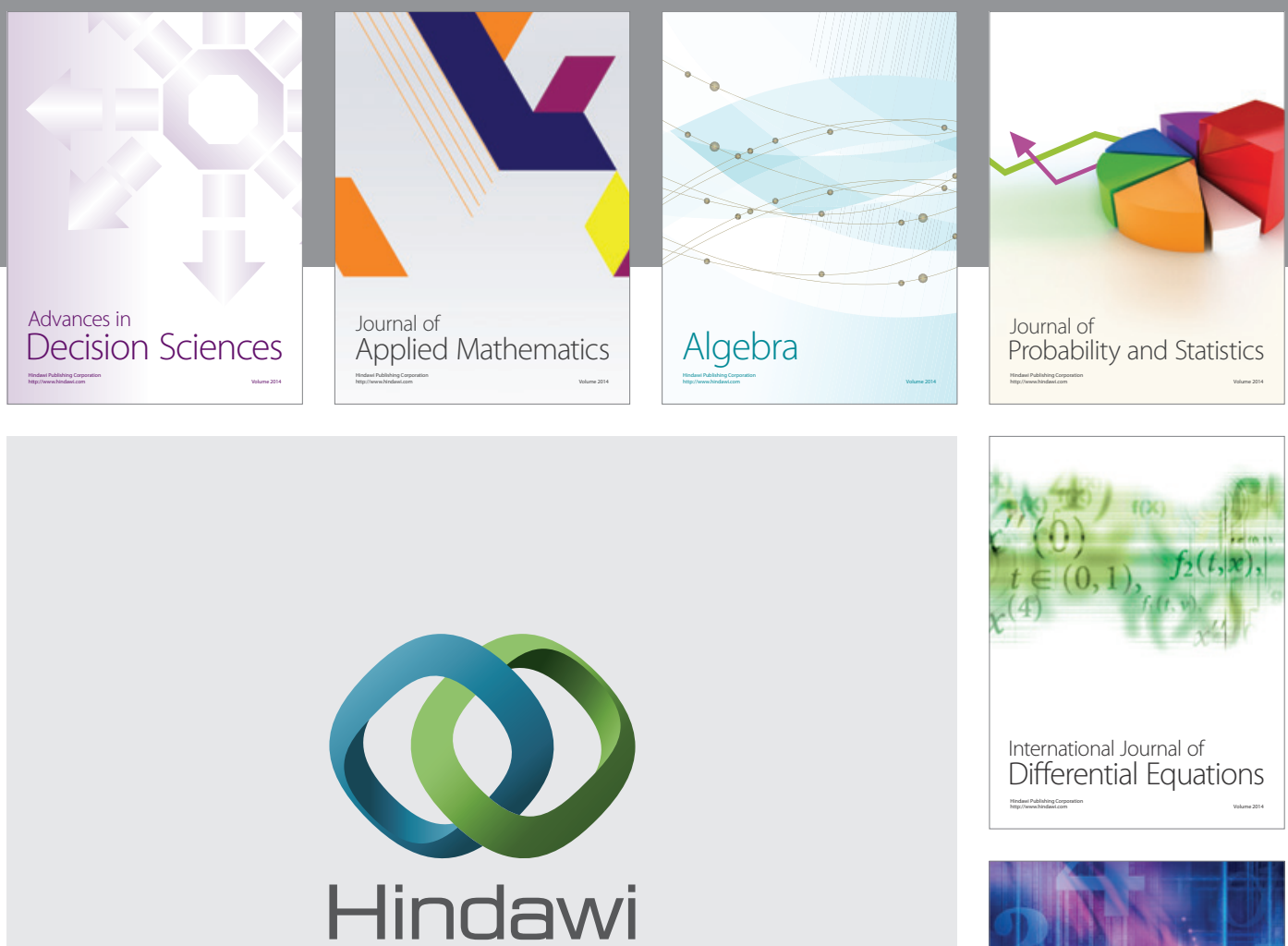

Submit your manuscripts at http://www.hindawi.com
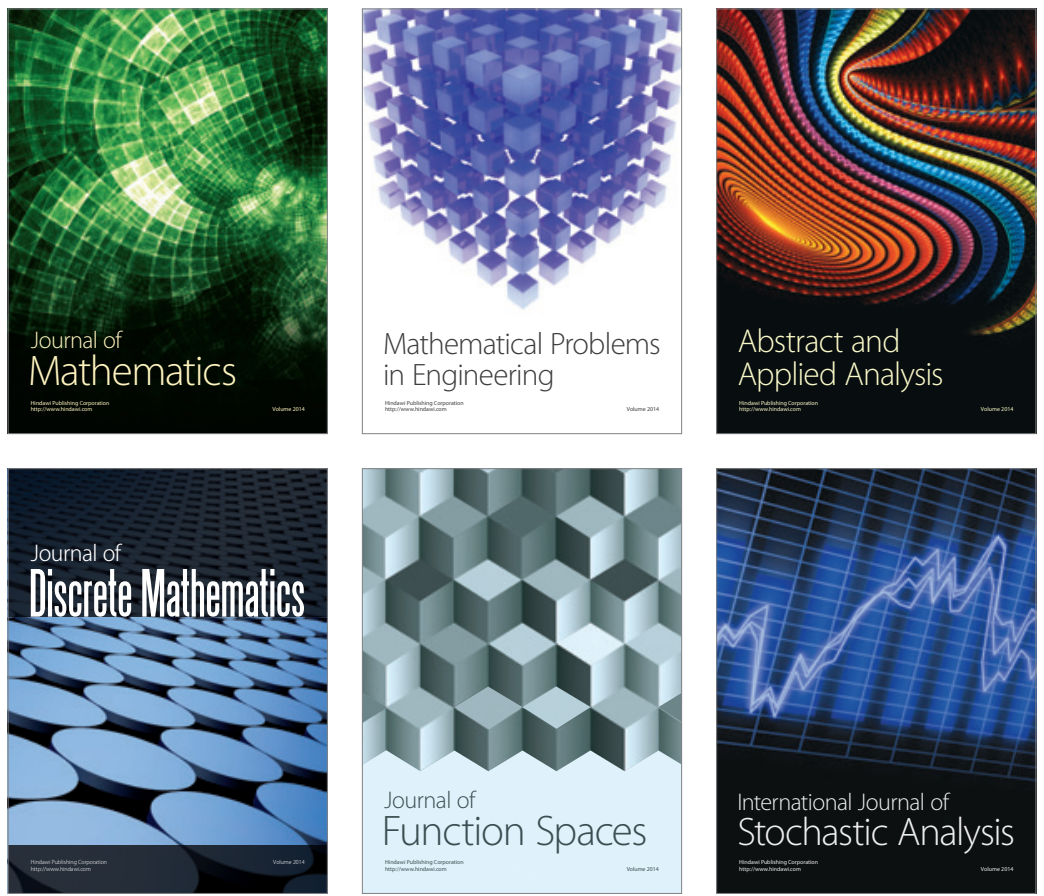

Journal of

Function Spaces

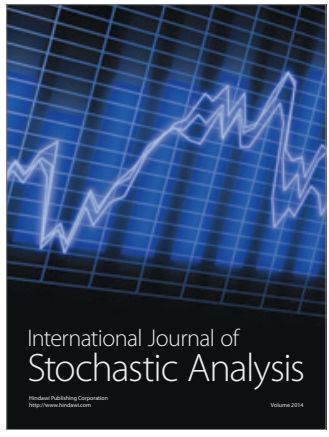

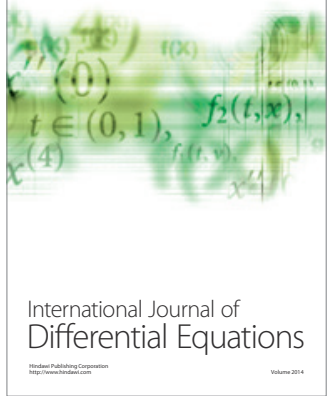
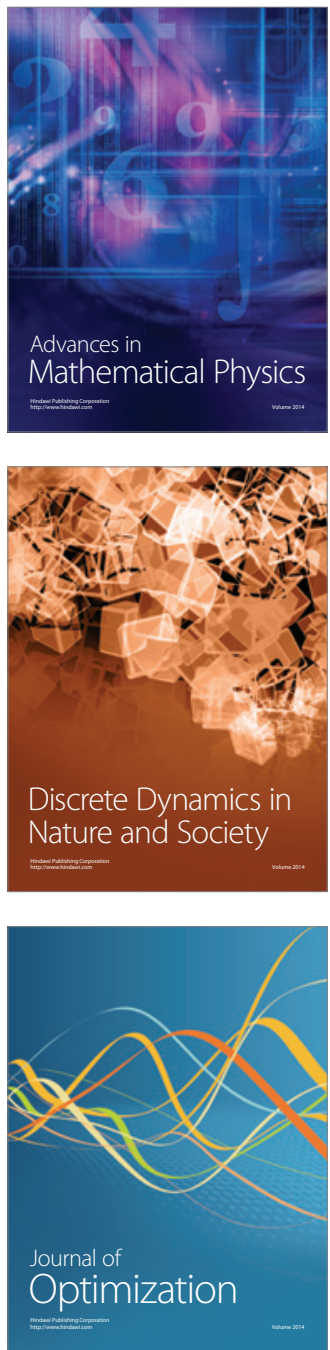